\title{
Orientational effects in electric-field-induced second-harmonic generation
}

\author{
David L Andrews and Brad S Sherborne \\ School of Chemical Sciences, University of East Anglia, Norwich, Norfolk NR4 7TJ, \\ England
}

Received 25 March 1986

\begin{abstract}
The theory of electric-field-induced second-harmonic generation is developed within the framework of quantum electrodynamics. Using a recently developed exact averaging procedure to account for the anisotropy introduced to a dipolar molecular fluid by the static electric field, the polarisation dependence of the process is investigated. $A$ number of experimental arrangements are suggested which allow a discrimination to be made between the two mechanisms involved: the third-order optical route facilitated by the field-induced anisotropy, and the fourth-order electro-optical route. This allows measurement of the corresponding non-linear optical and electro-optical tensor parameters. The full temperature and field-strength dependences are presented in detail, and it is shown that in two particular polarisation cases, that of helicity conversion, and where the static field and pump polarisation are perpendicular to the polarisation of the harmonic, the usual approximation for the orientational average is incomplete and produces misleading results.
\end{abstract}

\section{Introduction}

It is well known that second-harmonic generation (frequency doubling) is forbidden in isotropic media, regardless of the molecular symmetry. In fact it has been shown that this is true of all processes which generate even harmonics (Andrews 1980), even when all electric and magnetic multipolar interactions are considered. However, application of a static electric field to any dipolar molecular fluid introduces anisotropy, and thus allows second-harmonic generation to take place. Also, a higher-order electro-optical process is induced by the static field and contributes to the harmonic conversion even in non-polar, isotropic media. Thus electric-field-induced secondharmonic generation in any dipolar molecular fluid occurs by the combined action of two mechanisms, one induced directly by the static field, the other allowed by the field-induced anisotropy. The intense electric fields associated with highly intense laser beams can produce a similar effect (Mossberg et al 1978, Miyazaki et al 1981).

Electric-field-induced second-harmonic generation (EFISHG) was first observed in gases by Mayer (1968) and Hauchecorne et al (1971). Whilst the theory of the subject has been extensively treated previously (see for example Kielich 1969, 1970, 1979), recent theoretical advances now allow an exact treatment of the anisotropic averages required. In this paper, by treating the process within the framework of quantum electrodynamics (QED), using an exact averaging procedure, expressions are derived for the intensity of harmonic emission in terms of molecular tensors. Temperature, field and polarisation dependences are explicitly given in the results, whilst the 
frequency dependence is implicit in the definitions of the molecular tensors. Only two approximations are made: the QED treatment is in the electric dipole approximation and the dilute-gas approximation is used.

\section{Quantum formalism}

The starting point for the calculation is the quantum-electrodynamical Hamiltonian for the system, comprising the molecules and the electromagnetic radiation, modified to incorporate the effect of the static electric field. This may be expressed as

$$
H=\sum_{\tau} H_{\text {mol }}^{\prime}(\tau)+\sum_{\tau} H_{\mathrm{int}}(\tau)+H_{\mathrm{rad}}
$$

where $H_{\text {mol }}^{\prime}(\tau)$ is the Schrödinger operator for a molecule labelled $\tau$ in the presence of a static electric field $\boldsymbol{E}, H_{\mathrm{int}}(\tau)$ represents the interaction of this molecule with the electromagnetic radiation field, and $H_{\mathrm{rad}}$ is the Hamiltonian for the radiation. The sum in equation (1) is taken over all molecules in the laser beam. The process of harmonic generation may then be formulated in terms of time-dependent perturbation theory with the dressed eigenstates of $H_{\text {mol }}^{\prime}(\tau)$ and $H_{\mathrm{rad}}$ as a basis and $H_{\mathrm{int}}(\tau)$ as the perturbation. The appropriate dressed molecular eigenstates are given by

$$
\left|r_{\tau}^{\prime}\right\rangle=\left|r_{\tau}\right\rangle-\sum_{s \neq r}\left[\boldsymbol{\mu}^{s r}(\tau) \cdot \boldsymbol{E}\right] E_{r s}^{-1}\left|s_{\tau}\right\rangle+\ldots
$$

where $\left|r_{\tau}\right\rangle$ represents an eigenstate of the conventional Schrödinger operator $H_{\text {mol }}(\tau)$ for the molecule $\tau$ in the absence of an applied field, $E_{r s}$ denotes the difference $E_{r}^{(0)}-E_{s}^{(0)}$ between the zeroth-order energies associated with the states $\left|r_{\tau}\right\rangle$ and $\left|s_{\tau}\right\rangle$, and $\boldsymbol{\mu}^{s r}(\tau)$ is the transition electric dipole moment for the $\left|s_{\tau}\right\rangle \leftarrow\left|r_{\tau}\right\rangle$ transition. In writing equation (2) it has been assumed that the electric field $\boldsymbol{E}$ is not only temporally but also spatially homogeneous across the laser beam.

Within the electric dipole approximation the interaction Hamiltonian may be written as

$$
H_{\mathrm{int}}(\tau)=-\varepsilon_{0}^{-1} \mu(\tau) \cdot d^{\perp}\left(\boldsymbol{R}^{\tau}\right)
$$

where $\boldsymbol{\mu}(\tau)$ is the electric dipole moment operator for molecule $\tau$ located at $\boldsymbol{R}^{\tau}$ and $\boldsymbol{d}^{\perp}\left(\boldsymbol{R}^{\tau}\right)$ is the transverse electric displacement operator for the radiative field, which has the mode expansion (Craig and Thirunamachandran 1984)

$$
\begin{gathered}
\boldsymbol{d}^{\perp}(\boldsymbol{R})=\mathrm{i} \sum_{\boldsymbol{k}, \lambda}\left(h c k \varepsilon_{0} / 2 V\right)^{1 / 2}\left[\boldsymbol{e}^{\lambda}(\boldsymbol{k}) a^{\lambda}(\boldsymbol{k}) \exp (\mathrm{i} \boldsymbol{k} \cdot \boldsymbol{R})\right. \\
\left.-\overline{\boldsymbol{e}}^{\lambda}(\boldsymbol{k}) \bar{a}^{\dagger \lambda}(\boldsymbol{k}) \exp (-\mathrm{i} \boldsymbol{k} \cdot \boldsymbol{R})\right] .
\end{gathered}
$$

Here the sum is over the transverse modes of the radiation with wavevector $\boldsymbol{k}$ (circular frequency $\omega=c|k|$ ) and polarisation vector $e^{\lambda}(\boldsymbol{k})$, associated with annihilation and creation operators $a^{\lambda}(\boldsymbol{k})$ and $a^{\dagger \lambda}(\boldsymbol{k})$ respectively, and $V$ is the quantisation volume.

For the derivation of the harmonic power the matrix element $M_{\mathrm{fi}}$ connecting the initial and final states of the radiation field must be evaluated. By neglecting wavefunction overlap this may be expressed as a sum of contributions $M_{\mathrm{fi}}^{\tau}$ from each molecule:

$$
M_{\mathrm{fi}}=\sum_{\tau} M_{\mathrm{fi}}^{\tau}
$$


where each term has the usual perturbation series expansion

$$
\begin{aligned}
& M_{\mathrm{fi}}^{\tau}=\left\langle\mathrm{f}_{\mathrm{s}}^{\tau}\left|H_{\mathrm{int}}\right| \mathbf{i}_{\mathrm{s}}^{\tau}\right\rangle+\sum_{\mathrm{I}_{\mathrm{s}}}^{\prime} \frac{\left\langle\mathrm{f}_{\mathrm{s}}^{\tau}\left|H_{\text {int }}\right| \mathbf{I}_{\mathrm{s}}^{\tau}\right\rangle\left\langle\mathbf{I}_{\mathrm{s}}^{\tau}\left|H_{\mathrm{int}}\right| \mathrm{i}_{\mathrm{s}}^{\tau}\right\rangle}{E\left(\mathrm{i}_{\mathrm{s}}^{\tau}\right)-E\left(\mathbf{I}_{\mathrm{s}}^{\tau}\right)} \\
& +\sum_{\mathbf{i}_{\mathrm{s}}, \mathrm{I}_{\mathrm{s}}} \frac{\left\langle\mathrm{f}_{\mathrm{s}}^{\tau}\left|H_{\mathrm{int}}\right| \mathrm{II}_{\mathrm{s}}^{\tau}\right\rangle\left\langle\mathrm{II}_{\mathrm{s}}^{\tau}\left|H_{\mathrm{int}}\right| \mathrm{I}_{\mathrm{s}}^{\tau}\right\rangle\left\langle\mathrm{I}_{\mathrm{s}}^{\tau}\left|H_{\mathrm{int}}\right| \mathrm{i}_{\mathrm{s}}^{\tau}\right\rangle}{\left[E\left(\mathrm{i}_{\mathrm{s}}^{\tau}\right)-E\left(\mathrm{II}_{\mathrm{s}}^{\tau}\right)\right]\left[E\left(\mathrm{i}_{\mathrm{s}}^{\tau}\right)-E\left(\mathrm{I}_{\mathrm{s}}^{\tau}\right)\right]} \\
& +\sum_{\mathrm{I}_{\mathrm{s}}, \mathrm{I}_{\mathrm{s}}, \mathrm{II} \mathrm{I}_{\mathrm{s}}}^{\prime} \frac{\left\langle\mathrm{f}_{\mathrm{s}}^{\tau}\left|H_{\mathrm{int}}\right| \mathrm{III}_{\mathrm{s}}^{\tau}\right\rangle\left\langle\mathrm{III}_{\mathrm{s}}^{\tau}\left|H_{\mathrm{int}}\right| \mathrm{II}_{\mathrm{s}}^{\tau}\right\rangle\left\langle\mathrm{II}_{\mathrm{s}}^{\tau}\left|H_{\mathrm{int}}\right| \mathrm{I}_{\mathrm{s}}^{\tau}\right\rangle\left\langle\mathrm{I}_{\mathrm{s}}^{\tau}\left|H_{\mathrm{int}}\right| \mathrm{i}_{\mathrm{s}}^{\tau}\right\rangle}{\left[\left(\mathrm{I}_{\mathrm{s}}^{\tau}\right)\right]\left[E\left(\mathrm{i}_{\mathrm{s}}^{\tau}\right)-E\left(\mathrm{II}_{\mathrm{s}}^{\tau}\right)\right]\left[E\left(\mathrm{i}_{\mathrm{s}}^{\tau}\right)-E\left(\mathrm{I}_{\mathrm{s}}^{\tau}\right)\right]}+\ldots
\end{aligned}
$$

In equation (6) the subscript s denotes the fact that both states and energies relate to the system comprising the molecule $\tau$ plus the radiation. The primes denote omission of the initial and final states of the system from the summation over intermediate states, which excludes the permanent moments from the optical and electro-optical tensors.

For second-harmonic generation at a centre $\tau$ in its dressed ground state, $\left|0_{\tau}^{\prime}\right\rangle$, the initial and final states of the system are given by

$$
\begin{aligned}
& \left|\mathrm{i}_{\tau}^{\mathrm{s}}\right\rangle=\left|0_{\tau}^{\prime}\right\rangle|n(\boldsymbol{k}, \boldsymbol{e})\rangle \\
& \left|\mathrm{f}_{\tau}^{\mathrm{s}}\right\rangle=\left|0_{\tau}^{\prime}\right\rangle|(\boldsymbol{n}-2)(\boldsymbol{k}, \boldsymbol{e})\rangle\left|1\left(\boldsymbol{k}^{\prime}, \boldsymbol{e}^{\prime}\right)\right\rangle
\end{aligned}
$$

where $\boldsymbol{k}$ and $\boldsymbol{e}$ are the wavenumber and polarisation vectors of the pump beam and $\boldsymbol{k}^{\prime}$ and $\boldsymbol{e}^{\prime}$ are the corresponding vectors for harmonic emission, with the requirement that $2 k=k^{\prime}$. The numbers in the kets give the occupation numbers of the corresponding modes, all other modes being empty.

The leading contributions to equation (6) are

$$
\begin{aligned}
M_{\mathrm{fi}}^{\tau}=-\frac{1}{2} \mathrm{i}(\hbar \omega / & \left.V \varepsilon_{0}\right)^{3 / 2} n^{1 / 2}(n-1)^{1 / 2}\left[\beta_{i(j k)}^{\tau}(-2 \omega ; \omega, \omega) \bar{e}_{i}^{\prime} e_{j} e_{k}\right. \\
& \left.+\chi_{i(j k) l}^{\tau}(-2 \omega ; \omega, \omega, 0) \bar{e}_{i}^{\prime} e_{j} e_{k} E_{l}+\ldots\right] \exp \left[\mathrm{i}\left(2 \boldsymbol{k}-\boldsymbol{k}^{\prime}\right) \cdot \boldsymbol{R}^{\tau}\right]
\end{aligned}
$$

with implied summation over repeated tensor indices. In this equation $\beta_{i(j k)}^{\tau}$ is the molecular hyperpolarisability tensor of molecule $\tau$, which is symmetric in the last two indices, and $\chi_{i(j k) l}^{\tau}$ is the fourth-rank non-linear susceptibility tensor, symmetric in its middle two indices. These are defined as

$$
\begin{aligned}
\beta_{i(j k)}=\frac{1}{2} \sum_{r, s}( & \left.\frac{\mu_{i}^{0 s} \mu_{j}^{s r} \mu_{k}^{r 0}}{\left(E_{r 0}-\hbar \omega\right)\left(E_{s 0}-2 \hbar \omega\right)}+\frac{\mu_{j}^{0 s} \mu_{i}^{s r} \mu_{k}^{r 0}}{\left(E_{r 0}-\hbar \omega\right)\left(E_{s 0}+\hbar \omega\right)}+\frac{\mu_{j}^{0 s} \mu_{k}^{s r} \mu_{i}^{r 0}}{\left(E_{r 0}+2 \hbar \omega\right)\left(E_{s 0}+\hbar \omega\right)}\right) \\
& +\frac{1}{2}(j \leftrightarrow k) \\
\chi_{i(j k) l}=\frac{1}{2} \sum_{r, s, t}( & \frac{\mu_{i}^{0 t} \mu_{l}^{t s} \mu_{j}^{s r} \mu_{k}^{r 0}}{\left(E_{r 0}-\hbar \omega\right)\left(E_{s 0}-2 \hbar \omega\right)\left(E_{t 0}-2 \hbar \omega\right)} \\
& +\frac{\mu_{i}^{0 t} \mu_{k}^{t s} \mu_{l}^{s r} \mu_{j}^{r 0}}{\left(E_{r 0}-\hbar \omega\right)\left(E_{s 0}-\hbar \omega\right)\left(E_{t 0}-2 \hbar \omega\right)}+\frac{\mu_{i}^{0 t} \mu_{k}^{t s} \mu_{j}^{s r} \mu_{l}^{r 0}}{E_{r 0}\left(E_{s 0}-\hbar \omega\right)\left(E_{t 0}-2 \hbar \omega\right)} \\
& +\frac{\mu_{l}^{0 t} \mu_{i}^{t s} \mu_{k}^{s r} \mu_{j}^{r 0}}{\left(E_{r 0}-\hbar \omega\right)\left(E_{s 0}-2 \hbar \omega\right) E_{t 0}}+\frac{\mu_{l}^{0 t} \mu_{k}^{t s} \mu_{i}^{s r} \mu_{j}^{r 0}}{\left(E_{r 0}-\hbar \omega\right)\left(E_{s 0}+\hbar \omega\right) E_{t 0}} \\
& +\frac{\mu_{l}^{0 t} \mu_{k}^{t s} \mu_{j}^{s r} \mu_{i}^{r 0}}{\left(E_{r 0}+2 \hbar \omega\right)\left(E_{s 0}+\hbar \omega\right) E_{t 0}}+\frac{\mu_{k}^{0 t} \mu_{i}^{t s} \mu_{l}^{s r} \mu_{j}^{r 0}}{\left(E_{r 0}-\hbar \omega\right)\left(E_{s 0}-\hbar \omega\right)\left(E_{t 0}+\hbar \omega\right)}
\end{aligned}
$$




$$
\begin{aligned}
& +\frac{\mu_{k}^{0 t} \mu_{i}^{t s} \mu_{i}^{s r} \mu_{j}^{r 0}}{\left(E_{r 0}-\hbar \omega\right)\left(E_{s 0}+\hbar \omega\right)\left(E_{t 0}+\hbar \omega\right)}+\frac{\mu_{k}^{0 t} \mu_{l}^{t s} \mu_{j}^{s r} \mu_{i}^{r 0}}{\left(E_{r 0}+2 \hbar \omega\right)\left(E_{s 0}+\hbar \omega\right)\left(E_{t 0}+\hbar \omega\right)} \\
& +\frac{\mu_{k}^{0 t} \mu_{j}^{t s} \mu_{l}^{s r} \mu_{i}^{r 0}}{\left(E_{r 0}+2 \hbar \omega\right)\left(E_{s 0}+2 \hbar \omega\right)\left(E_{t 0}+\hbar \omega\right)}+\frac{\mu_{k}^{0 t} \mu_{i}^{t s} \mu_{j}^{s r} \mu_{l}^{r 0}}{E_{r 0}\left(E_{s 0}-\hbar \omega\right)\left(E_{r 0}+\hbar \omega\right)} \\
& \left.+\frac{\mu_{k}^{0 t} \mu_{j}^{t s} \mu_{i}^{s r} \mu_{l}^{r 0}}{E_{r 0}\left(E_{s 0}+2 \hbar \omega\right)\left(E_{t 0}+\hbar \omega\right)}\right)+\frac{1}{2}(j \leftrightarrow k) .
\end{aligned}
$$

It should be noted that whereas the $\beta$ tensor can be identically zero, as for example in the case of centrosymmetric molecules, $\chi$ is always finite in the same way as the molecular polarisability.

The identical result is obtained by explicit inclusion of the static field interaction in the interaction Hamiltonian, in contrast to the 'dressed molecule' approach used above. The equivalence between these two approaches has recently been discussed in more detail in connection with electric-field-induced absorption (Andrews and Sherborne 1984).

Using equation (9), and ignoring higher-order terms, gives

$$
\left|M_{\mathrm{fi}}\right|^{2}=\frac{\hbar^{3} \omega^{3}}{4 V^{3} \varepsilon_{0}^{3}} n(n-1)\left|\sum_{\tau}\left(\beta_{i(j k)}^{\tau} \bar{e}_{i}^{\prime} e_{j} e_{k}+\chi_{i(j k)}^{\tau} \bar{e}_{i}^{\prime} e_{j} e_{k} E_{l}\right) \exp \left[\mathrm{i}\left(2 \boldsymbol{k}-\boldsymbol{k}^{\prime}\right) \cdot \boldsymbol{R}^{\tau}\right]\right|^{2}
$$

which can be expanded as

$$
\begin{aligned}
\left|M_{\mathrm{fi}}\right|^{2}=\frac{\hbar^{3} \omega^{3}}{4 V^{3} \varepsilon_{0}^{3}} & n(n-1)\left(\sum_{\tau}\left(\beta_{i(j k)}^{\tau} \bar{e}_{i}^{\prime} e_{j} e_{k}+\chi_{i(j k) l}^{\tau} \bar{e}_{i}^{\prime} e_{j} e_{k} E_{l}\right)\right. \\
& \left.\times \sum_{\tau^{\prime}}\left(\bar{\beta}_{i(j k)}^{\tau^{\prime}} e_{i}^{\prime} \bar{e}_{j} \bar{e}_{k}+\bar{\chi}_{i(j k) l}^{\tau^{\prime}} e_{i}^{\prime} \bar{e}_{j} \bar{e}_{k} E_{l}\right)\right) \exp \left[\mathrm{i}\left(2 k-\boldsymbol{k}^{\prime}\right) \cdot \boldsymbol{R}^{\tau \tau^{\prime}}\right]
\end{aligned}
$$

where

$$
\boldsymbol{R}^{\tau \tau^{\prime}}=\boldsymbol{R}^{\tau}-\boldsymbol{R}^{\tau^{\prime}}
$$

There are then two separable contributions, the incoherent diagonal term where $\tau=\tau^{\prime}$, and the coherent off-diagonal term where $\tau \neq \tau^{\prime}$. The incoherent term gives the hyper-Rayleigh contribution (Terhune et al 1965, Kielich et al 1971, 1974) and corresponds to a relatively weak background scattering in comparison with the total harmonic power. Dropping the incoherent term, and then using the fact that, irrespective of any orientational order, the gross translational invariance of the system ensures that the spatial and orientational factors can be separated, we obtain

$$
\left|M_{\mathrm{fi}}\right|^{2}=\frac{\hbar^{3} \omega^{3}}{4 V^{3} \varepsilon_{0}^{3}} n(n-1)\left|\left\langle\beta_{i(j k)} \bar{e}_{i}^{\prime} e_{j} e_{k}+\chi_{i(j k) l} \bar{e}_{i}^{\prime} e_{j} e_{k} E_{l}\right\rangle\right|^{2} \sum_{\tau} \sum_{\tau^{\prime} \neq \tau} \exp \left[\mathrm{i}\left(2 \boldsymbol{k}-\boldsymbol{k}^{\prime}\right) \cdot \boldsymbol{R}^{\tau \tau^{\prime}}\right]
$$

where the angular brackets denote rotational averaging to allow for the distribution of molecular orientations. The summation over the spatial distribution can be written

$$
\begin{aligned}
& \sum_{\tau} \sum_{\tau^{\prime} \neq \tau} \exp \left[\mathrm{i}\left(2 k-\boldsymbol{k}^{\prime}\right) \cdot \boldsymbol{R}^{\tau \tau^{\prime}}\right] \\
& =\frac{N(N-1)}{V^{2}} \iint \exp \left[\mathrm{i}\left(2 \boldsymbol{k}-\boldsymbol{k}^{\prime}\right) \cdot \boldsymbol{R}^{\tau \tau^{\prime}}\right] F_{2}\left(\boldsymbol{R}^{\tau}, \boldsymbol{R}^{\tau^{\prime}}\right) \mathrm{d} \boldsymbol{R}^{\prime \prime} \mathrm{d} \boldsymbol{R}^{\tau^{\prime}}
\end{aligned}
$$


where $N$ is the number of molecules in the interaction volume, and $F_{2}\left(\boldsymbol{R}^{\tau}, \boldsymbol{R}^{\tau \prime}\right)$ is the conditional probability density function (Zimm 1945). In the dilute-gas approximation, contributions from short-range order are negligible and equation (15) gives

$\left|M_{\mathrm{fi}}\right|^{2}=\frac{\hbar^{3} \omega^{3}}{4 V^{3} \varepsilon_{0}^{3}} n(n-1) N(N-1) \pi \delta\left(2 k-k^{\prime}\right)\left|\left\langle\beta_{i(j k)} \bar{e}_{i}^{\prime} e_{j} e_{k}+\chi_{i(j k) l} \bar{e}_{i}^{\prime} e_{j} e_{k} E_{l}\right)\right|^{2}$.

The Dirac delta function in the above equation highlights the fact that coherent emission is in the forward direction. Using time-dependent perturbation theory the emitted harmonic power $\phi$ can now be written

$$
\phi(t)=\sum_{k^{\prime}} \frac{2 k^{\prime}\left|M_{\mathrm{fi}}\right|^{2}}{\hbar} \frac{\sin \left[c\left(k^{\prime}-2 k\right) t\right]}{\left(k^{\prime}-2 k\right)}
$$

where $t$ is the elapsed time. On converting the summation to an integral and using the properties of the delta function in equation (17), we obtain the continuous-wave (cw) solution

$$
\phi=\frac{\hbar^{2} c^{3} k^{6}}{2 V^{2} \pi^{2} \varepsilon_{0}^{3}} n(n-1) N(N-1)\left|\left\langle\beta_{i(j k)} \bar{e}_{i}^{\prime} e_{j} e_{k}+\chi_{i(j k) l} \bar{e}_{i}^{\prime} e_{j} e_{k} E_{l}\right\rangle\right|^{2} .
$$

This expression is strictly applicable only where the incident radiation is a number state. However, the coherence and statistical properties of the laser beam can be incorporated into the theory by adopting a more general representation in terms of linear combinations of such number states, and the corresponding result for the harmonic radiant power is then

$$
\phi=\frac{\bar{I}_{0}^{2} g^{(2)} \omega^{4}}{2 \pi^{2} \varepsilon_{0}^{3} c^{5}} N(N-1)\left|\left\langle\beta_{i(j k)} \bar{e}_{i}^{\prime} e_{j} e_{k}+\chi_{i(j k) l} \bar{e}_{i}^{\prime} e_{j} e_{k} E_{l}\right\rangle\right|^{2}
$$

where $\bar{I}_{0}$ is the mean irradiance of the pump beam and $g^{(2)}$ is its degree of second-order coherence (Loudon 1983).

\section{Boltzmann-weighted orientational averages}

In general, the rotational average in equation (20) has to be effected with regard to an anisotropic distribution of molecular orientations. This results from the preferential alignment of the polar molecules with the applied static field, determined by the torque $\boldsymbol{\mu}^{00} \times \boldsymbol{E}$, where $\boldsymbol{\mu}^{00}$ is the static electric dipole moment. Any molecular orientation by interaction with the electric field of the laser beam will be negligible.

By weighting the averages in equation (20) with the Boltzmann term $\exp \left(\boldsymbol{\mu}^{00} \cdot \boldsymbol{E} / k T\right)$ to allow for the anisotropic distribution, the following expression for the harmonic power is obtained:

$\phi=K\left|\left\langle\left(\beta_{i(j k)} \bar{e}_{i}^{\prime} e_{j} e_{k}+\chi_{i(j k) l} \bar{e}_{i}^{\prime} e_{j} e_{k} E_{l}\right) \exp \left(\boldsymbol{\mu}^{00} \cdot \boldsymbol{E} / k T\right)\right\rangle\left\langle\exp \left(\boldsymbol{\mu}^{00} \cdot \boldsymbol{E} / k T\right)\right\rangle^{-1}\right|^{2}$

where for large $N$

$$
K \simeq \frac{\bar{I}_{0}^{2} g^{(2)} N^{2} \omega^{4}}{2 \pi^{2} c^{5} \varepsilon_{0}^{3}}
$$

The weighted average in equation $(21)$ is traditionally approximated by expanding the exponential and retaining only the linear terms (see for example Chemla and Bonneville 
1978, Kielich 1979). However, it has recently been shown that an exact result in terms of spherical Bessel functions can be obtained by referring all molecular parameters to a molecule-fixed Cartesian frame (denoted by Greek indices) and averaging over the direction cosines between axes in the laboratory-fixed and molecule-fixed frames (Andrews and Harlow 1984). Thus if $l_{i \lambda}$ denotes the direction cosine between the $i$ axis in the laboratory frame and the $\lambda$ axis in the molecular frame, the expression becomes

$$
\begin{aligned}
& \phi=K \mid\left[\beta_{\lambda(\mu \nu)} \bar{e}_{i}^{\prime} e_{j} e_{k}\left\langle l_{i \lambda} l_{j \mu} l_{k \nu} \exp \left(\mu_{\rho}^{00} E_{n} l_{n \rho} / k T\right)\right\rangle\right. \\
&+ \\
&\left.+\chi_{\lambda(\mu \nu) o} \bar{e}_{i}^{\prime} e_{j} e_{k} E_{l}\left\langle l_{i \lambda} l_{j \mu} l_{k \nu} l_{l o} \exp \left(\mu_{\rho}^{00} E_{n} l_{n \rho} / k T\right)\right\rangle\right] \\
& \times\left.\left\langle\exp \left(\mu_{\sigma}^{00} E_{\rho} l_{p \sigma} / k T\right)\right\rangle^{-1}\right|^{2}
\end{aligned}
$$

which can be expressed as

$$
\begin{aligned}
\phi=K \mid\left[\beta_{\lambda(\mu \nu)} \overline{\boldsymbol{e}}_{i}^{\prime} e_{j} e_{k} I_{i j k ; \lambda \mu \nu}^{(3) \phi}\left(-\mathrm{i} \gamma, \hat{\boldsymbol{E}}, \hat{\boldsymbol{\mu}}^{00}\right)\right. \\
\left.+\chi_{\lambda(\mu \nu) o} e_{i}^{\prime} e_{j} e_{k} E_{l} I_{i j k l ; \lambda \mu \nu \nu}^{(4) \phi}\left(-\mathrm{i} \gamma, \hat{\boldsymbol{E}}, \hat{\boldsymbol{\mu}}^{00}\right)\right]\left.\left[I^{(0) \phi}\left(-\mathrm{i} \gamma, \hat{\boldsymbol{E}}, \hat{\boldsymbol{\mu}}^{00}\right)\right]^{-1}\right|^{2}
\end{aligned}
$$

where

$$
\gamma=\mu^{00} E / k T
$$

and the results for the weighted averages $I^{(n) \phi}$ are given by Andrews and Harlow (1984).

\section{Results}

Invoking the results for the weighted averages in equation (24) leads to the following expression for the harmonic power with arbitrary beam polarisation and field direction:

$$
\begin{aligned}
\phi=K \mid\left\{\frac{1}{5} \mathrm{i}_{1}(-\mathrm{i} \gamma)\right. & {\left[\left(3 p_{1}-p_{2}\right) \beta_{2}+\left(2 p_{2}-p_{1}\right) \beta_{1}\right]-j_{2}(-\mathrm{i} \gamma) p_{4} \beta_{3} } \\
& -\frac{1}{10} \mathrm{i} j_{3}(-\mathrm{i} \gamma)\left[\left(5 p_{3}-2 p_{1}-p_{2}\right)\left(5 \beta_{4}-2 \beta_{2}-\beta_{1}\right)\right] \\
& +\frac{1}{15} E j_{0}(-\mathrm{i} \gamma)\left[\left(3 p_{1}-p_{2}\right) \chi_{1}+\left(2 p_{2}-p_{1}\right) \chi_{2}\right]+\frac{1}{5} \mathrm{i} E j_{1}(-\mathrm{i} \gamma)\left[\left(\chi_{3}+\chi_{4}\right) p_{4}\right] \\
& -\frac{1}{14} E j_{2}(-\mathrm{i} \gamma)\left[2\left(5 p_{1}-2 p_{3}-p_{2}\right) \chi_{8}+\left(5 p_{3}-2 p_{1}-p_{2}\right)\left(\chi_{7}+2 \chi_{5}\right)\right. \\
& \left.+2\left(3 p_{2}-p_{1}-p_{3}\right) \chi_{6}+2\left(3 p_{1}+3 p_{3}-2 p_{2}\right) \chi_{1}+\left(5 p_{2}+3 p_{3}-4 p_{1}\right) \chi_{2}\right] \\
& -\frac{1}{5} \mathrm{i} E j_{3}(-\mathrm{i} \gamma)\left[\left(5 \chi_{9}-\chi_{3}-\chi_{4}\right) p_{4}\right]+\frac{1}{70} E j_{4}(-\mathrm{i} \gamma)\left[\left(5 p_{3}-2 p_{1}-p_{2}\right)\right. \\
& \left.\left.\times\left(35 \chi_{10}-10 \chi_{8}-5 \chi_{7}-5 \chi_{6}-10 \chi_{5}+2 \chi_{1}+\chi_{2}\right)\right]\right\}\left.j_{0}^{-1}(-\mathrm{i} \gamma)\right|^{2}
\end{aligned}
$$

\begin{tabular}{|c|c|c|c|}
\hline $\begin{array}{l}\chi_{1}=\chi_{\lambda(\lambda \mu) \mu} \\
\chi_{S}=\chi_{\mu(\lambda \nu) \lambda} \hat{\mu}_{\mu} \hat{\mu}_{\nu} \\
\chi_{9}=\chi_{\lambda(\nu \mu) O} \hat{\mu}_{o} \hat{\mu}_{\tau} \hat{\mu}_{\nu} \varepsilon_{\lambda \mu \tau}\end{array}$ & $\begin{array}{l}\chi_{2}=\chi_{\lambda(\mu \mu) \lambda} \\
\chi_{6}=\chi_{\mu(\lambda \lambda) \nu} \hat{\mu}_{\mu} \hat{\mu}_{\nu} \\
\chi_{10}=\chi_{\lambda(\mu \nu) 0} \hat{\mu}_{\lambda} \hat{\mu}_{\mu} \hat{\mu}_{\nu} \hat{\mu}_{o}\end{array}$ & $\begin{array}{l}\chi_{3}=\chi_{\lambda(\mu \nu) o} \hat{\mu}_{\mu} \varepsilon_{\lambda \nu o} \\
\chi_{7}=\chi_{\lambda(\mu \nu) \lambda} \hat{\mu}_{\mu} \hat{\mu}_{\nu}\end{array}$ & $\begin{array}{l}\chi_{4}=\chi_{\lambda(\mu \nu) \nu} \hat{\mu}_{\tau} \varepsilon_{\lambda \mu \tau} \\
\chi_{8}=\chi_{\lambda(\lambda \mu) \nu} \hat{\mu}_{\mu} \hat{\mu}_{\nu}\end{array}$ \\
\hline$\beta_{1}=\beta_{\lambda \mu \mu} \hat{\mu}_{\lambda}$ & $\beta_{2}=\beta_{\lambda \lambda \mu} \hat{\mu}_{\mu}$ & $\beta_{3}=\beta_{\lambda \mu \nu} \hat{\mu}_{\nu} \hat{\mu}_{\tau} \varepsilon_{\lambda \mu \tau}$ & $\beta_{4}=\beta_{\lambda \mu \nu} \hat{\mu}_{\lambda} \hat{\mu}_{\mu} \hat{\mu}_{\nu}$ \\
\hline$p_{1}=\left(\overline{\boldsymbol{e}}^{\prime} \cdot \boldsymbol{e}\right)(\boldsymbol{e} \cdot \hat{\boldsymbol{E}})$ & $p_{2}=\left(\overline{\boldsymbol{e}}^{\prime} \cdot \hat{\boldsymbol{E}}\right)(\boldsymbol{e} \cdot \boldsymbol{e})$ & $p_{3}=\left(\overline{\boldsymbol{e}}^{\prime} \cdot \hat{\boldsymbol{E}}\right)(\boldsymbol{e} \cdot \hat{\boldsymbol{E}})^{2}$ & $p_{4}=\left(\overline{\boldsymbol{e}}^{\prime} \times \boldsymbol{e}\right) \cdot \hat{\boldsymbol{E}}(\boldsymbol{e} \cdot \hat{\boldsymbol{E}})$ \\
\hline
\end{tabular}

where $p_{1}$ to $p_{4}$ are polarisation parameters with values dictated by experimental conditions and $\beta_{1}$ to $\beta_{4}, \chi_{1}$ to $\chi_{10}$ are molecular invariants as defined in table 1 . If the $\beta$ and $\chi$ tensors are assumed to be fully index symmetric, as in the Kleinman

Table 1. Molecular and polarisation parameters involved in the theory of EFISHG. 
treatment (Kleinman 1962, Franken and Ward 1963), then we have the equalities $\beta_{1}=\beta_{2}, \beta_{3}=0, \chi_{1}=\chi_{2}, \chi_{5}=\chi_{6}=\chi_{7}=\chi_{8}, \chi_{3}=\chi_{4}=\chi_{9}=0$. However full index symmetry can only arise when both the pump and harmonic frequencies are well removed from all absorption bands.

It is pertinent to note here that $p_{1}$ to $p_{4}$ all cancel if the static electric field is collinear with the direction of beam propagation, $z$. Thus, it is henceforth assumed that the static field is in the transverse $x$ direction, in the same $x y$ plane as the polarisation vectors $e$ and $e^{\prime}$. In table 2 the values of the polarisation parameters are given for 16 distinct experimental arrangements.

In table 3 the functional form for the reduced spherical Bessel functions, $j_{n}^{\prime}(-\mathrm{i} \gamma)=$ $j_{n}(-\mathrm{i} \gamma) / j_{0}(-\mathrm{i} \gamma)$, are detailed. In most cases, the static electric field will be sufficiently small to allow the low-field limit $\gamma \ll 1$ to be applicable, which allows the use of a Taylor series expansion for $\operatorname{coth}(\gamma)$. For completeness the high-field limit where $\operatorname{coth}(\gamma) \simeq 1$ is also included.

Before demonstrating the detailed use of these results, some general comments specific to the pump polarisation are made.

\subsection{Plane-polarised pump}

Reference to table 2 immediately shows that with a plane-polarised pump the harmonic beam is completely polarised parallel to the direction of the applied static field.

Table 2. The polarisation parameters as a function of beam and detector polarisations, in the case where the static field $E$ is directed along the $x$ direction, transverse to the beam propagation direction $z$. Only the non-zero parameters are included in the table.

\begin{tabular}{|c|c|c|c|c|c|}
\hline & & \multicolumn{4}{|c|}{ Pump polarisation } \\
\hline & & $x$ & $y$ & $\mathrm{~L}$ & $\mathrm{R}$ \\
\hline $\begin{array}{l}\text { Harmonic } \\
\text { polarisation } \\
\text { component }\end{array}$ & $\begin{array}{l}x \\
y \\
\mathrm{~L} \\
\mathrm{R}\end{array}$ & $\begin{array}{l}p_{1}=p_{2}=p_{3}=1 \\
p_{1}=p_{2}=p_{3}=2^{-1 / 2} \\
p_{1}=p_{2}=p_{3}=2^{-1 / 2}\end{array}$ & $\begin{array}{l}p_{2}=1 \\
p_{2}=2^{-1 / 2} \\
p_{2}=2^{-1 / 2}\end{array}$ & $\begin{array}{l}p_{1}=p_{3}=\frac{1}{2} \\
p_{1}=\frac{1}{2} \mathrm{i} \\
p_{1}=2^{-1 / 2}, p_{3}=8^{-1 / 2} \\
p_{3}=8^{-1 / 2}\end{array}$ & $\begin{array}{l}p_{1}=p_{3}=\frac{1}{2} \\
p_{1}=\frac{1}{2} \mathrm{i} \\
p_{3}=8^{-1 / 2} \\
p_{1}=2^{-1 / 2}, p_{3}=8^{-1 / 2}\end{array}$ \\
\hline
\end{tabular}

Table 3. Analytic form and asymptotic limits of the reduced spherical Bessel functions, $j_{n}^{\prime}=j_{n} / j_{0}$.

\begin{tabular}{lll}
\hline Full expression for $j_{n}^{\prime}(-\mathrm{i} \gamma)$ & Low-field limit, $\gamma \ll 1$ & High-field limit, $\gamma \gg 1$ \\
\hline$j_{0}^{\prime}=1$ & $j_{0}^{\prime}=1$ & $j_{0}^{\prime}=1$ \\
$j_{1}^{\prime}=\mathrm{i}\left(\frac{1}{\gamma}-\operatorname{coth}(\gamma)\right)$ & $j_{1}^{\prime}=-\mathrm{i}\left(\frac{\gamma}{3}-\frac{\gamma^{3}}{45}+\frac{2 \gamma^{5}}{945}+\ldots\right)$ & $j_{1}^{\prime}=\mathrm{i}\left(\frac{1}{\gamma}-1\right)$ \\
$j_{2}^{\prime}=-1-\frac{3}{\gamma^{2}}+\frac{3}{\gamma} \operatorname{coth}(\gamma)$ & $j_{2}^{\prime}=-\frac{\gamma^{2}}{15}+\frac{2 \gamma^{4}}{315}+\ldots$ & $j_{2}^{\prime}=-1+\frac{3}{\gamma}-\frac{3}{\gamma^{2}}$ \\
$j_{3}^{\prime}=\mathrm{i}\left[\left(1+\frac{15}{\gamma^{2}}\right) \operatorname{coth}(\gamma)-\frac{6}{\gamma}-\frac{15}{\gamma^{3}}\right]$ & $j_{3}^{\prime}=\mathrm{i}\left(\frac{\gamma^{3}}{105}-\frac{\gamma^{5}}{945}+\ldots\right)$ & $j_{3}^{\prime}=\mathrm{i}\left(1-\frac{6}{\gamma}+\frac{15}{\gamma^{2}}-\frac{15}{\gamma^{3}}\right)$ \\
$j_{4}^{\prime}=1+\frac{45}{\gamma^{2}}+\frac{105}{\gamma^{4}}-\left(\frac{10}{\gamma}+\frac{105}{\gamma^{3}}\right) \operatorname{coth}(\gamma)$ & $j_{4}^{\prime}=\frac{\gamma^{4}}{945}+\ldots$ & $j_{4}^{\prime}=1-\frac{10}{\gamma}+\frac{45}{\gamma^{2}}-\frac{105}{\gamma^{3}}+\frac{105}{\gamma^{4}}$ \\
\hline
\end{tabular}


Denoting the emission power for pump polarisation $\lambda$ and harmonic polarisation $\mu$ by $\phi_{\lambda}^{\mu}$, the total harmonic power can be expressed as

$$
\phi_{\lambda}^{\text {total }}=\phi_{\lambda}^{\mathrm{L}}+\phi_{\lambda}^{\mathrm{R}}=\phi_{\lambda}^{x}+\phi_{\lambda}^{y} .
$$

From table 2, the following identities also hold:

$$
\begin{aligned}
& \phi_{\lambda}^{x}=2 \phi_{\lambda}^{\mathrm{L}}=2 \phi_{\lambda}^{\mathrm{R}} \\
& \phi_{\lambda}^{y}=0
\end{aligned}
$$

from which it is obvious that no helicity is introduced by the emission process. Inclusion of higher-order magnetic dipole and electric quadrupole terms in the interaction Hamiltonian does allow a weak chiral discrimination to be manifest, however (see for example Lam and Thirunamachandran 1982).

\subsection{Circularly polarised pump}

In the absence of a static field it has been shown that the generation of harmonics from a circularly polarised pump is forbidden for all even harmonics (Tang and Rabin 1971, Andrews 1980); however, in the presence of a static electric field, this rule is relaxed. Indeed, if the harmonic is resolved for its polarisation component in the $y$ direction, then only a circularly polarised pump can produce a measurable signal.

In the case of helicity conversion it becomes particularly transparent that this effect arises from the anisotropy, as can be seen by substituting the results from table 2 in equation (26). Since only the parameter $p_{3}$ is non-zero, the power expression takes the form

$$
\begin{gathered}
\phi=\frac{1}{196} K \mid-7 \mathrm{i} j_{3}^{\prime}\left(5 \beta_{4}-2 \beta_{2}-\beta_{1}\right)-E j_{2}^{\prime}\left(-4 \chi_{8}+5 \chi_{7}-2 \chi_{6}+10 \chi_{5}+3 \chi_{2}+6 \chi_{1}\right) \\
+E j_{4}^{\prime}\left(35 \chi_{10}-10 \chi_{8}-5 \chi_{7}-5 \chi_{6}-10 \chi_{5}+\chi_{2}+2 \chi_{1}\right)^{2}
\end{gathered}
$$

where each reduced spherical Bessel function has $(-i \gamma)$ as its argument. In the low-field limit, the leading term in equation (30) will be of $E^{6}, T^{-4}$ dependence. None of these terms appear if the standard approximation is made of retaining only the linear term in the expansion of the Boltzmann weighting exponential.

\section{Discussion}

A number of recent experiments have been performed in an attempt to separate the $\beta$ and $\chi$ tensor components (see for example Pantinakis et al 1985, Dudley and Ward 1985). With the data provided in tables 1-3 and the master equation (26) it is possible to devise a series of experiments that will enable the experimental evaluation of such molecular parameters. Also the relative importance of the two mechanisms responsible for EFISHG in a dipolar fluid can be investigated in this way.

At the extreme low-field limit, terms above first order in $\gamma$ may be ignored, giving from equation (26) and table 3

$$
\phi=\frac{K E^{2}}{225}\left|\left[\left(3 p_{1}-p_{2}\right)\left(\chi_{1}+\frac{\mu}{k T} \beta_{2}\right)+\left(2 p_{2}-p_{1}\right)\left(\chi_{2}+\frac{\mu}{k T} \beta_{1}\right)\right]\right|^{2} \text {. }
$$


If the field geometry is as in figure 1 , with $\psi=45^{\circ}$, experimental arrangements can be devised as follows:

$$
\begin{array}{rlll}
\left(3 p_{1}-p_{2}\right)=0 & \rightarrow & \tan \theta \tan \psi=\frac{1}{2}, \quad \text { so } \quad \theta=-63.4^{\circ} \\
\left(2 p_{2}-p_{1}\right)=0 & \rightarrow & \tan \theta \tan \psi=-2, \quad \text { so } \quad \theta=26.6^{\circ}
\end{array}
$$

giving

$$
\begin{aligned}
& \phi=\frac{K E^{2}}{90}\left(\chi_{2}^{2}+2 \frac{\mu}{k T} \beta_{1} \chi_{2}+\ldots\right) \\
& \phi=\frac{K E^{2}}{90}\left(\chi_{1}^{2}+2 \frac{\mu}{k T} \beta_{2} \chi_{1}+\ldots\right) .
\end{aligned}
$$

Hence, selective temperature experiments in these two geometries will lead directly to values for the four electro-optical parameters $\chi_{1}, \chi_{2}, \beta_{1}$ and $\beta_{2}$. Such parameters provide useful information on molecular electronic properties, such as the extent of delocalisation in conjugated species (Flytzanis 1979).

The individual components of the tensors $\beta$ and $\chi$, such as $\chi_{x x x x}$ and $\chi_{x x y y}$, cannot in general be experimentally determined simply by choice of beam polarisation and field direction as suggested by Hameka and Svendsen (1984). Whilst such an approach would be feasible in a solid sample, the distribution of molecular orientations in any fluid system results in the harmonic emission being determined only by the trace parameters such as $\chi_{\lambda(\lambda \mu) \mu}$. Of course, for molecules of high symmetry, the number of independent components of $\chi$ is greatly reduced, and individual components may then be determined through use of the relationships which hold between them (Reintjes 1984).

Another useful experimental arrangement is to constrain the geometry such that of all the polarisation parameters only $p_{4}$ is non-zero. This necessitates a static field with a finite component in the beam propagation direction, in contrast to the transverse direction considered above. A suitable arrangement is given in figure 2, and leads to the result that

$$
\phi=\frac{K E^{4}}{900}\left(\frac{\mu^{2}}{k^{2} T^{2}}\left(\chi_{3}+\chi_{4}\right)^{2}+\frac{2 \mu^{3}}{k^{3} T^{3}}\left(\chi_{3}+\chi_{4}\right) \beta_{3}+\ldots\right) \sin ^{2} 2 \psi
$$

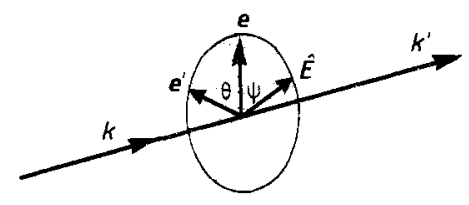

Figure 1. Configuration of polarisation vectors with a transverse static field for EFISHG with a plane-polarised pump.

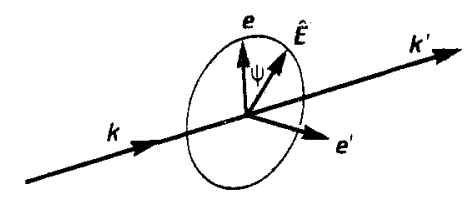

Figure 2. EFISHG configuration in which the harmonic is analysed for a polarisation component perpendicular to the pump polarisation, and the electric field lies in the plane containing the $(e, k)$ directions. 
where in this case the electro-optical parameters $\chi_{3}+\chi_{4}$ and $\beta_{3}$ can be directly measured. Once again, the non-zero emission with a fourth-power dependence on the field in this particular configuration is a feature which would not be expected from a theory based on the conventional expansion of the Boltzmann weighting.

In conclusion, it has been demonstrated that the usual approximation for the orientational average in EFISHG is incomplete, and for certain polarisation configurations leads to misleading results. In addition, the detailed polarisation measurements described above facilitate the determination of a number of characteristic parameters. These should prove useful for assessing the properties of new materials, for refining molecular wavefunction calculations and for generally increasing the number of parameters in terms of which the non-linear optical and electro-optical properties of molecules can be described.

\section{Acknowledgment}

We acknowledge with thanks the award of a SERC Studentship to BSS.

\section{References}

Andrews D L 1980 J. Phys. B: At. Mol. Phys. 134091

Andrews D L and Harlow M J 1984 Phys. Rev. A 292796

Andrews D L and Sherborne B S 1984 Chem. Phys. 881

Chemla D S and Bonneville R 1978 J. Chem. Phys. 682214

Craig D P and Thirunamachandran T 1984 Molecular Quantum Electrodynamics (New York: Academic)

Dudley J W II and Ward J F 1985 J. Chem. Phys. 824673

Flytzanis C 1979 Nonlinear Behaviour of Molecules, Atoms and Ions in Electric, Magnetic or Electromagnetic Fields ed L Neel (Amsterdam: Elsevier) p 185

Franken P A and Ward J F 1963 Rev. Mod. Phys. 3523

Hameka H F and Svendsen E N 1984 Int. J. Quantum Chem. Symp. 18525

Hauchecorne G, Kerhervé F and Mayer G 1971 J. Physique 3247

Kielich S 1969 IEEE J. Quantum Electron. QE-5 562

1970 Opto-electronics 25

1979 Nonlinear Behaviour of Molecules, Atoms and Ions in Electric, Magnetic or Electromagnetic Fields ed L Neel (Amsterdam: Elsevier) p 111

Kielich S, Kozierowski M, Ozgo Z and Zawodny R 1974 Acta Phys. Polon. A 459

Kielich S, Lalanne J R and Martin F B 1971 Phys. Rev. Lett. 261295

Kleinman D A 1962 Phys. Rev. 1291977

Lam Y T and Thirunamachandran T 1982 J. Chem. Phys. 773810

Loudon R 1983 The Quantum Theory of Light 2nd edn (Oxford: Clarendon)

Mayer G 1968 C. R. Acad. Sci. Paris B 26754

Miyazaki K, Sato T and Kashiwagi H 1981 Phys. Rev. A 231358

Mossberg T, Flusberg A and Hartmann S R 1978 Opt. Commun. 25121

Pantinakis A, Dean K J and Buckingham A D 1985 Chem. Phys. Lett. 120135

Reintjes J F 1984 Nonlinear Optical Parametric Processes in Liquids and Gases (New York: Academic)

Tang C L and Rabin H 1971 Phys. Rev. B 34025

Terhune R W, Maker P D and Savage C M 1965 Phys. Rev. Lett. 14681

Zimm B H 1945 J. Chem. Phys. 13141 